\title{
GEOS-Chem High Performance (GCHP v11-02c): a next-generation implementation of the GEOS-Chem chemical transport model for massively parallel applications
}

\author{
Sebastian D. Eastham ${ }^{1,2}$, Michael S. Long ${ }^{2}$, Christoph A. Keller ${ }^{3,4}$, Elizabeth Lundgren ${ }^{2}$, Robert M. Yantosca ${ }^{2}$, \\ Jiawei Zhuang ${ }^{2}$, Chi Li ${ }^{5}$, Colin J. Lee ${ }^{5}$, Matthew Yannetti ${ }^{2}$, Benjamin M. Auer ${ }^{3,6}$, Thomas L. Clune ${ }^{3}$, \\ Jules Kouatchou ${ }^{3,6}$, William M. Putman ${ }^{3}$, Matthew A. Thompson ${ }^{3,6}$, Atanas L. Trayanov ${ }^{3,6}$, Andrea M. Molod ${ }^{3}$, \\ Randall V. Martin ${ }^{5,7}$, and Daniel J. Jacob ${ }^{2}$ \\ ${ }^{1}$ Laboratory for Aviation and the Environment, Massachusetts Institute of Technology, Cambridge, Massachusetts, USA \\ ${ }^{2}$ John A. Paulson School of Engineering and Applied Sciences, Harvard University, Cambridge, Massachusetts, USA \\ ${ }^{3}$ NASA Global Modeling and Assimilation Office, Greenbelt, Maryland, USA \\ ${ }^{4}$ Universities Space Research Association, Columbia, Maryland, USA \\ ${ }^{5}$ Department of Physics and Atmospheric Science, Dalhousie University, Halifax, Nova Scotia, Canada \\ ${ }^{6}$ Science Systems and Applications, Inc., Lanham, Maryland, USA \\ ${ }^{7}$ Smithsonian Astrophysical Observatory, Harvard-Smithsonian Center for Astrophysics, Cambridge, Massachusetts, USA
}

Correspondence: Sebastian D. Eastham (seastham@mit.edu)

Received: 26 February 2018 - Discussion started: 8 March 2018

Revised: 28 June 2018 - Accepted: 11 July 2018 - Published: 24 July 2018

\begin{abstract}
Global modeling of atmospheric chemistry is a grand computational challenge because of the need to simulate large coupled systems of $\sim 100-1000$ chemical species interacting with transport on all scales. Offline chemical transport models (CTMs), where the chemical continuity equations are solved using meteorological data as input, have usability advantages and are important vehicles for developing atmospheric chemistry knowledge that can then be transferred to Earth system models. However, they have generally not been designed to take advantage of massively parallel computing architectures. Here, we develop such a highperformance capability for GEOS-Chem (GCHP), a CTM driven by meteorological data from the NASA Goddard Earth Observation System (GEOS) and used by hundreds of research groups worldwide. GCHP is a grid-independent implementation of GEOS-Chem using the Earth System Modeling Framework (ESMF) that permits the same standard model to operate in a distributed-memory framework for massive parallelization. GCHP also allows GEOS-Chem to take advantage of the native GEOS cubed-sphere grid for greater accuracy and computational efficiency in simulat-
\end{abstract}

ing transport. GCHP enables GEOS-Chem simulations to be conducted with high computational scalability up to at least 500 cores, so that global simulations of stratospheretroposphere oxidant-aerosol chemistry at $\mathrm{C} 180$ spatial resolution $\left(\sim 0.5^{\circ} \times 0.625^{\circ}\right)$ or finer become routinely feasible.

\section{Introduction}

Atmospheric chemistry models are used to address a wide range of problems related to climate forcing, air quality, and atmospheric deposition. Simulations of oxidant and aerosol chemistry involve hundreds of chemically interacting species, coupled to transport on all scales. The computational demands are considerable, which has limited the inclusion of atmospheric chemistry in climate models (National Research Council, 2012). Offline chemical transport models (CTMs), where meteorology is provided as input data from a parent global climate model (GCM) or atmospheric data assimilation system (DAS), are frequently used for rea- 
sons of simplicity, reproducibility, and ability to focus on chemical processes. The global GEOS-Chem CTM originally described by Bey et al. (2001), using meteorological input from the Goddard Earth Observation System (GEOS) DAS of the NASA Global Modeling and Assimilation Office (GMAO), is used by hundreds of atmospheric chemistry research groups worldwide (http://www.geos-chem.org, last access: 19 July 2018). Increasing computational resources in the form of massively parallel architectures can allow GEOSChem users to explore more complex problems at higher grid resolutions, but this requires re-engineering of the model to take advantage of these architectures. Here, we describe a high-performance version of GEOS-Chem (GCHP) engineered for this purpose, and we demonstrate its ability to access a new range of capability and scales for global atmospheric chemistry modeling.

The original GEOS-Chem CTM (GEOS-Chem Classic, or GCC) was designed for shared-memory (OpenMP) parallelization. A detailed description of the model including a user manual is available on the GEOS-Chem website (http: //www.geos-chem.org, last access: 19 July 2018). Computation is distributed over a number of cores on a single node, with data held in shared arrays. But recent growth in computational power has taken the form of massively parallel networked systems, where additional computational power is achieved by increasing the number of identical nodes rather than by improving the nodes themselves. This has placed a restriction on growth in the problem size and complexity which can be solved by a single instance of GCC. To take advantage of massively parallel architectures, a new framework is needed which allows GEOS-Chem to use a distributedmemory model, where the computation is distributed across multiple coordinated nodes using a Message Passing Interface (MPI) implementation such as MVAPICH2 or OpenMPI.

An important first step in this evolution was the integration of GEOS-Chem as the online chemistry component within the GEOS DAS (Long et al., 2015). In order to ensure that the online and offline versions of GEOS-Chem were identical, GCC was modified to use the exact same code in the independent CTM and in the DAS. Major modifications were required to make GEOS-Chem grid-independent and compatible with the GEOS Modeling and Analysis Prediction Layer (MAPL) (Suarez et al., 2007), an Earth System Modeling Framework (ESMF) (Hill et al., 2004) based software layer which handles communication between different components of the GEOS DAS. The GEOS-Chem code was adapted to accept an arbitrarily sized horizontal set of atmospheric columns, with no requirements regarding adjacency of the columns or overall coverage of any particular set. All these changes were made "under the hood" in the standard GEOS-Chem code. When GEOS-Chem is run as GCC, the set of columns is designated as a single block which covers the entire globe or a subset in a nested domain, and parallelization is achieved by internally running parallel loops over the columns. When GEOS-Chem is run as part of GEOS, MAPL internally splits the atmosphere into smaller domains, each of which contains a different set of atmospheric columns. These domains can then be distributed across multiple nodes, exploiting massively parallel architectures. As a result of these changes, the same GEOS-Chem code can now be run either as a stand-alone, shared-memory offline CTM, or as a GCM component in the massively parallel, distributed GEOS DAS. Any improvement in chemical modeling developed for the offline CTM is thus immediately available in the GEOS DAS version, which never becomes out of date and remains referenceable to the current version of GEOS-Chem.

In this work, we take the next logical step of developing GCHP as a distributed-memory, MAPL-based implementation of the GEOS-Chem CTM. GCHP uses an identical copy of the GEOS-Chem Classic (GCC) code to provide the same high-fidelity atmospheric chemical simulation capabilities, allowing users to switch between GCC and GCHP implementations with confidence that they are using the same model. The exact same internal code is used in GCC sharedmemory and GCHP distributed-memory applications. This closes the development loop between online and offline modeling. By sharing infrastructure code between GCHP and GEOS in the form of MAPL, offline modelers can now take advantage of modeling advances which originate in the online model in the same way that GEOS benefits from advances in chemical modeling developed in the GEOS-Chem CTM (Nielsen et al., 2017). By way of example, GEOS was recently able to conduct a full-year $13 \mathrm{~km}$ resolution "nature run" with the current standard version of GEOS-Chem tropospheric chemistry (Hu et al., 2018). In return, GCHP incorporates the more efficient cubed-sphere grid and the Finite-Volume Cubed-Sphere Dynamical Core (FV3) advection code present in GEOS, and is capable of directly ingesting GEOS output in its native cubed-sphere format.

\section{Model description}

\subsection{Overview}

Atmospheric chemistry models such as GEOS-Chem solve the 3-D chemical continuity equations for an ensemble of $m$ coupled chemical species (Brasseur and Jacob, 2017). The continuity equation for the number density $n_{i}$ (molecules $\mathrm{cm}^{-3}$ ) of species $i$ is expressed as

$\frac{\partial n_{i}}{\partial t}=-\nabla \cdot\left(n_{i} \boldsymbol{v}\right)+s_{i}$

where $v$ is the velocity vector $\left(\mathrm{m} \mathrm{s}^{-1}\right)$, and $s_{i}$ is the local net production and loss of species $i$ (molecules $\mathrm{cm}^{-3} \mathrm{~s}^{-1}$ ). In CTMs, $v$ is provided by archived output from a parent GCM or DAS, with subgrid-scale parameterized transport statistics (boundary layer mixing, deep convection) as additional CTM transport terms in Eq. (1). From a computational 


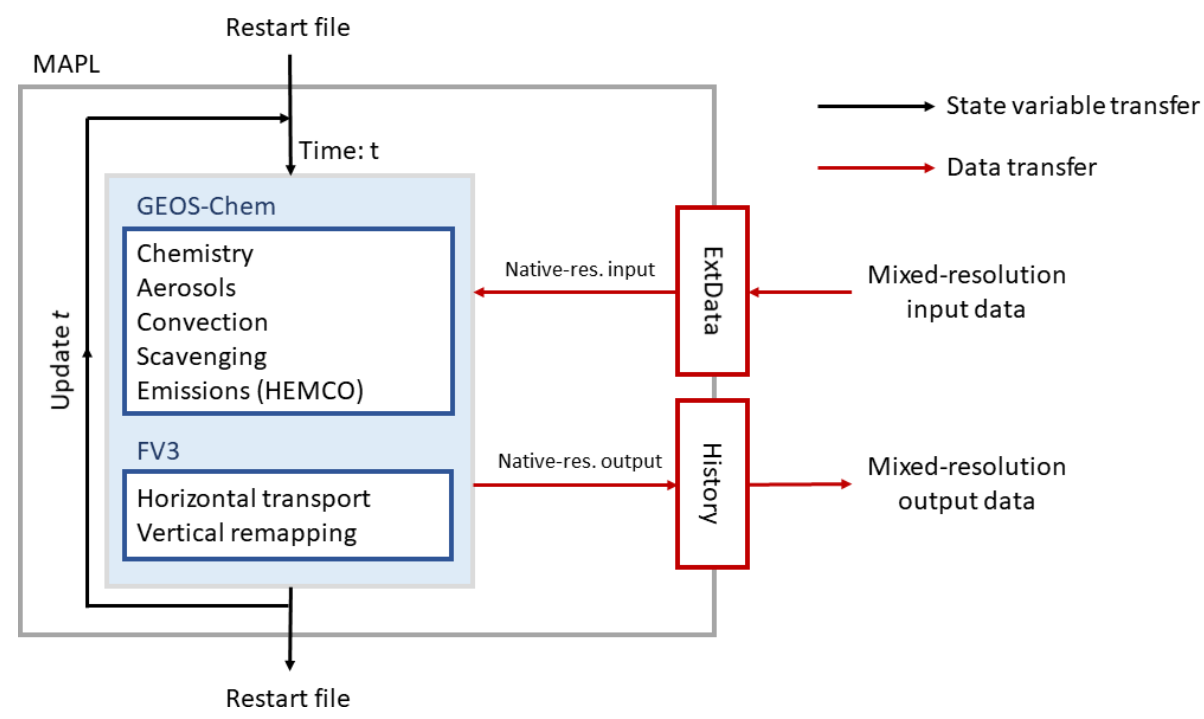

Figure 1. Connectivity of the major components of GCHP. The main time-stepping loop is represented by the feedback loop from the model output back into the input.

standpoint, the local term $s_{i}$ is grid-independent. However, the transport terms are grid-aware, as they move material between grid points. In GEOS-Chem, the atmosphere is split into independent columns, with each column made up of a number of discrete grid points (Long et al., 2015). Vertical processes (boundary layer mixing, deep convection) are then considered to be local in the sense that they are calculated independently for each column. In each column simulated by GEOS-Chem, the local term computes chemical evolution with a unified tropospheric-stratospheric mechanism (Eastham et al., 2014; Sherwen et al., 2016), convective transport (Wu et al., 2007), boundary layer mixing (Lin and McElroy, 2010), radiative transfer and photolysis (Prather, 2012), wet scavenging (Liu et al., 2001), dry deposition (Wang et al., 1998), particle sedimentation (Fairlie et al., 2007), and emissions (Keller et al., 2014).

The GEOS DAS meteorological fields used for input to GEOS-Chem are produced on a gnomonic cubed-sphere grid (Putman and Lin, 2007) at a current horizontal resolution of $\mathrm{C} 720(\sim 13 \mathrm{~km} \times 13 \mathrm{~km})$, with output provided operationally on a rectilinear grid at a resolution of $0.25^{\circ} \times$ $0.3125^{\circ}$. Currently, global oxidant-aerosol simulations with GEOS-Chem are effectively limited to $2^{\circ} \times 2.5^{\circ}$ resolution due to the prohibitive memory and time requirements of running a more finely resolved simulation on a single node. In order to progress to finer resolutions, GEOS-Chem must be able to split the requirements for memory and computation across multiple nodes, and to ensure that communication between the different nodes is minimal and efficient. This is the role of GCHP.

\subsection{GCHP v11-02c model architecture}

The general software architecture of the GCHP model is shown in Fig. 1. A detailed description including a user manual is available on the GCHP web page of the GEOS-Chem website (http://www.geos-chem.org, last access: 19 July 2018). The GMAO-developed MAPL is included in the GCHP code download and is automatically built when compiling GCHP for the first time. MAPL initializes the model, establishes the atmospheric domain on each computational core, and handles model coordination and internal communication. Transport within and between each of the domains is calculated by the FV3 advection component. Within each atmospheric domain, local terms are calculated by a standard copy of the GEOS-Chem Classic code, embedded in the model as described by Long et al. (2015). This copy of the GEOS-Chem code is identical to that used in GEOS-Chem Classic, such that all processes other than advection which are simulated in GCC are simulated identically in GCHP. GCHP v11-02c as presented here uses GEOS-Chem v11-02c. The embedded copy of GEOS-Chem in GCHP is compiled without OpenMP shared-memory parallelization, resulting in a pure MPI implementation. Data input is handled through the External Data (ExtData) component, and output is handled through the History component. ExtData and History are structural components of MAPL (Long et al., 2015; Nielsen et al., 2017; Suarez et al., 2007).

At initialization, a gridded representation of the atmosphere is generated by MAPL from user-specified input. GCHP can operate on any horizontal grid supported by MAPL as long as an appropriate advection scheme is available. Currently, the standard advection scheme in GCHP is the Putman and Lin FV3 scheme, which operates on a 
cubed-sphere discretization, described in Sect. 2.2. The initial state of the model is determined from a restart file, read by MAPL directly. During this stage, all relevant input data are also read into memory through the ExtData module. Data at any grid resolution are read from NetCDF files in disk storage, and are regridded on the fly to the resolution at which the model is running. This allows data on either rectilinear latitude-longitude or gnomonic cubed-sphere grids to be read in without requiring offline preprocessing. Data can be regridded using bilinear interpolation (used for wind fields), or first-order mass-conservative regridding (used for emissions and all other meteorological data). Conservative regridding is achieved using "tile files" generated analytically with the Tempest tool (Ullrich and Taylor, 2015). Additional regridding techniques are also available for special cases such as handling categorical (e.g., surface type) data. All constant fields are read in once, at the start of the simulation. For all time-varying fields, ExtData holds two samples in memory at all times: the previous sample ("left bracket") and the upcoming sample ("right bracket"). All fields can either be held constant between samples or smoothly interpolated between the two brackets.

Output is performed through the History component. Fields which are defined as "exports" within GCHP are tracked continuously by the History component. Any export can be requested by the user by adding it to an output collection in the HISTORY.rc input file, as either an instantaneous and/or time-averaged output. At each time step, the History component will acquire the current value of the field for each requested diagnostic and store it either at the native resolution or, if requested by the user, perform online regridding to a rectilinear latitude-longitude grid. This allows the user to decide the appropriate spatial and temporal resolution for their simulation output, independent of the resolution at which the simulation itself is conducted. All diagnostic quantities which are available in gridded form in GEOS-Chem Classic are automatically defined as exports in GCHP.

\subsection{Grid discretization and transport}

In GCHP, the atmosphere is divided into independent atmospheric columns, with a subset of columns forming a single domain which is assigned to one of the computational cores. All local operations, such as chemistry, deposition, and emissions, are handled locally by components already present in the core GEOS-Chem code. The advection operator transfers mass between adjacent columns, requiring MPI-based data communication between them at domain boundaries. The amount and frequency of the communication depend on the chosen grid discretization and transport algorithm.

\subsubsection{Grid discretization}

GCHP inherits the equidistant gnomonic cubed-sphere grid discretization used by the GEOS DAS (Putman and Lin,
1

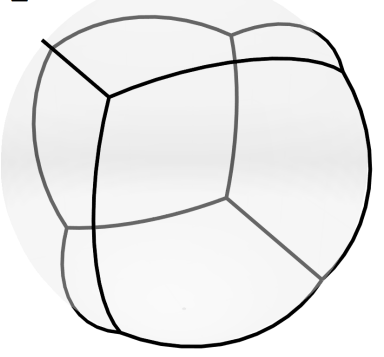

3

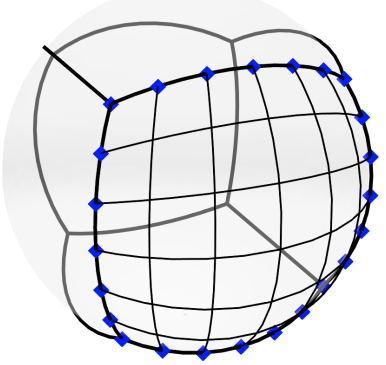

2

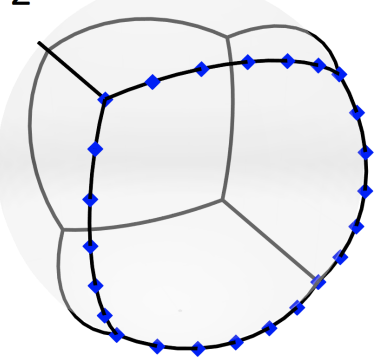

4

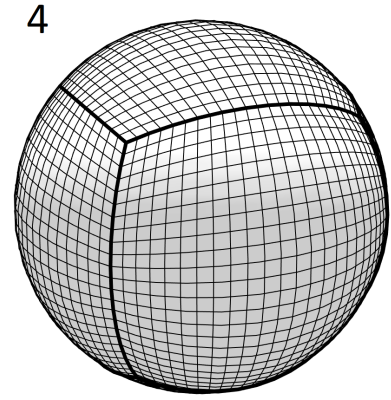

Figure 2. Graphical description of the process used to generate a gnomonic cubed-sphere grid. Subpanels are numbered based on the textual description of the steps. The grids shown are C6 and, on the final frame, C24. Demonstration is available interactively at http://www.geos-chem.org/cubed_sphere.html (last access: 19 July 2018).

2007). Cubed-sphere grids split the surface of a sphere into six equal-sized faces. Each face is then subdivided into cells of approximately equal size, with each cell representing an atmospheric column. The equidistant gnomonic projection splits each cube edge into $N$ equally sized segments, connecting the opposing edges with great circle arcs in order to generate a regular mesh (see Fig. 2). The grid resolution is referred to as $\mathrm{C} N$, such as $\mathrm{C} 48$ for a grid with $48 \times 48$ atmospheric columns on each of the six faces. The grid cell spacing is approximately $10000 / N \mathrm{~km}$, such that a C48 grid has a mean cell width of $\sim 200 \mathrm{~km}$. Each core is assigned a contiguous, rectangular set of columns on one of the six faces by MAPL, with the exact subdomain size determined based on the domain aspect ratio specified by the user at runtime.

Cubed-sphere grids offer several advantages over conventional rectilinear grids. The absolute cell size in a rectilinear grid decreases from the Equator to the poles, for structural rather than scientific reasons, resulting in larger CourantFriedrichs-Lewy (CFL) numbers at high latitudes. This reduces the minimum time step required for explicit Eulerian advection schemes to maintain stability. The problem can be mitigated by applying a semi-Lagrangian method when the CFL exceeds unity, at the expense of having to do nonphysical mass conservation corrections. In an MPI environment, these issues also complicate domain decomposition for the purposes of distributing the grid between cores. The use 
Table 1. Grid resolution and core counts for the performance test simulations. Both GCC and GCHP use v11-02c of the core GEOSChem code.

\begin{tabular}{llrr}
\hline $\begin{array}{l}\text { GEOS-Chem } \\
\text { implementation }\end{array}$ & $\begin{array}{l}\text { Grid resolution } \\
\text { resolution }\end{array}$ & $\begin{array}{r}\text { Number of } \\
\text { grid cells }\end{array}$ & $\begin{array}{r}\text { Number of } \\
\text { cores used }\end{array}$ \\
\hline GCHP & C24 & 250000 & $6-216$ \\
GCHP & C48 & 1000000 & $6-540$ \\
GCHP & C90 & 3500000 & $12-540$ \\
GCHP & C180 & 14000000 & $90-540$ \\
GCC & $4^{\circ} \times 5^{\circ}$ & 240000 & $6-30$ \\
GCC & $2^{\circ} \times 2.5^{\circ}$ & 940000 & $6-30$ \\
\hline
\end{tabular}

of a semi-Lagrangian scheme results in tracer mass being transferred between grid cells which are not considered to be adjacent, increasing the size of the halo for each domain and therefore the amount of communication necessary between cores.

The cubed-sphere grid helps to address these issues. The area ratio between the largest and smallest grid cells is $\sim 2.3$, regardless of the resolution. There are no polar singularities, although advection across the edges and corners of the cube requires special considerations.

Vertically, the atmosphere is discretized into hydrostatic, hybrid-sigma layers. The current GEOS DAS uses 72 layers ranging from the surface to $1 \mathrm{~Pa}$ at the upper edge.

\subsubsection{Transport}

Transport in GEOS-Chem is comprised of four operations: advection, moist convection, boundary layer mixing, and aerosol settling. The latter three operations occur purely incolumn and are unchanged between GCC and GCHP. However, advection must be grid aware. Horizontal advection in GCHP is calculated on a layer-by-layer basis using the cubed-sphere advection algorithm of Putman and Lin (2007). This algorithm is fourth-order accurate except at the six cube edges (2nd-order). Vertical advection is then calculated using a vertically Lagrangian method (Lin, 2004). Prior to the advection step, each core requests concentration data from neighboring domains to fill the halo region. Advection is then calculated independently for each atmospheric domain.

Horizontal mass fluxes and CFL numbers are either supplied directly to the model or are calculated based on $3 \mathrm{~h}$ average horizontal wind speed data and the instantaneous surface pressure at the start of the time step (time $t$ ). All fluxes are based on dry air mass and dry surface pressure. To ensure numerical stability, substepping is implemented such that the internal advection time step $\Delta t$ is subdivided into $n$ number of substeps until the CFL is less than 1. Horizontal advection is then performed $n$ times. If the number of substeps $n$ is greater than 1, changes to the air mass in each grid cell due to wind divergence are retained between substeps. The implied surface pressure resulting from changes in total column mass
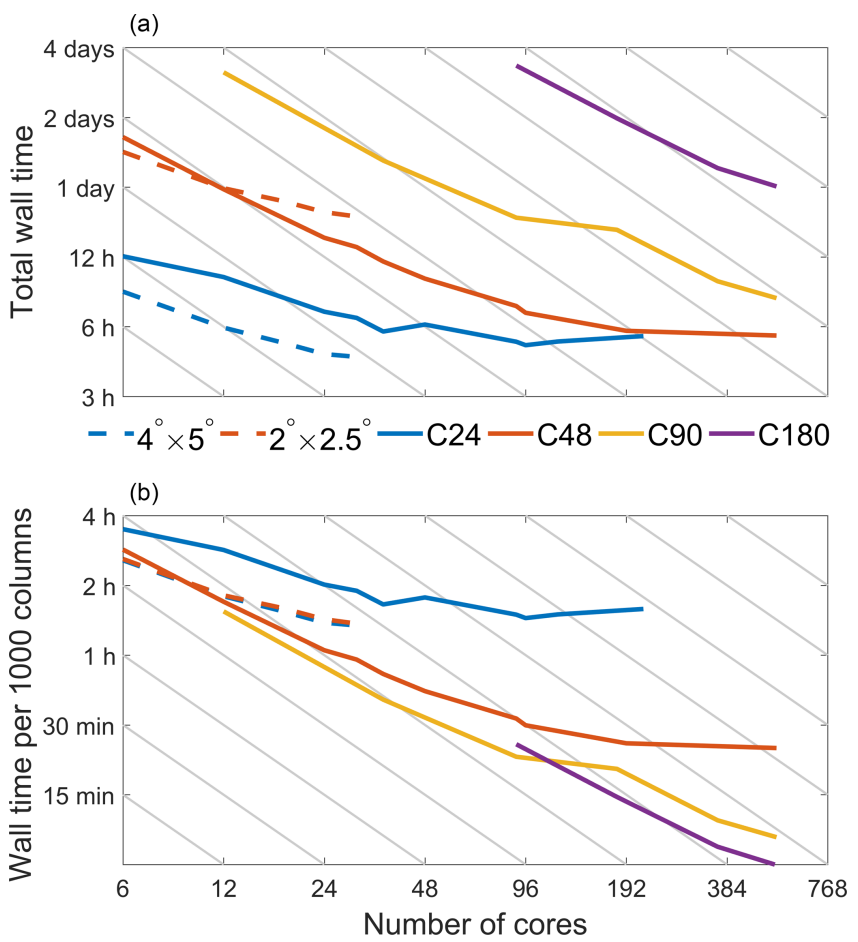

Figure 3. Wall time taken to perform 1-month GEOS-Chem simulations at different resolutions and with different numbers of cores. Panel (a) shows the absolute time taken to complete each simulation at each resolution. Panel (b) shows the wall time normalized by the number of atmospheric columns simulated at each resolution. Solid lines are for GCHP simulations (cubed-sphere grids) and dashed lines are for GCC simulations (latitude-longitude grids). Grey lines on each plot show perfect scaling, corresponding to a $50 \%$ reduction in runtime for each doubling of the number of cores.

is also updated. However, horizontal mass fluxes are assumed to be constant over the time step, and no vertical remapping is performed between substeps. When mass fluxes have to be estimated offline from wind data, the simulated pressure can diverge from that in the meteorological archive (Jöckel et al., 2001). GCC solves this problem with the pressure fixer of Horowitz et al. (2003), which modifies calculated air mass fluxes to ensure the correct surface pressure tendency based on zonal totals. However, this approach corrupts the horizontal transport to some extent, and a pressure fixer has not yet been designed for transport on the cubed sphere. For archives where air mass fluxes are not explicitly available, including the meteorological data used for this work, GCHP defaults to using a simple global air mass correction.

After the horizontal tracer advection loop is complete (time $t+\Delta t$ ), the total air mass in each vertical column will have changed, as will the vertical distribution. Vertical advection is calculated by remapping the deformed layers back to the hydrostatic hybrid-eta grid defined by the surface pressure, as interpolated from the meteorological archive for the 
post-advection time $(t+\Delta t)$. This ensures that the surface pressure accurately tracks that in the meteorological data.

\subsection{Benchmarking}

The standard benchmarking procedures applied to GEOSChem before each version release are also applied to GCHP, ensuring that the integrity of the model is maintained from version to version. Benchmarks involve a 1-year Unified Chemistry eXtension (UCX) (troposphere-stratosphere) oxidant-aerosol simulation with resolution of $4^{\circ} \times 5^{\circ}(\mathrm{GCC})$ or C48 (GCHP), plus a 1-year simulation of the ${ }^{222} \mathrm{Rn}-$ ${ }^{210} \mathrm{~Pb}-{ }^{7} \mathrm{Be}$ system (Liu et al., 2001) for updates that may affect transport. Further documentation of benchmark procedures is available at http://www.geos-chem.org (last access: 19 July 2018). Species concentrations and source/sink diagnostics from the benchmark simulation are archived and compared to the previous model version and to selected climatological data. Results are inspected by the model developers and by the GEOS-Chem Steering Committee, which gives final approval. There are small differences between GCHP and GCC benchmarks due to differences in transport algorithm, but otherwise the two functionalities perform identically.

\section{Model performance}

We analyzed the performance of GCHP v11-02c by conducting simulations at multiple grid resolutions (C24 to C180), each for a range of core counts (Table 1). For lowresolution applications, performance is also compared to the maximum achievable performance using the GCC v1102c shared-memory architecture. All simulations are for 1 month (July 2016) of troposphere-stratosphere oxidantaerosol chemistry, including 206 species and 135 tracers, and using operational meteorological data from GEOS forward processing (FP). The GCC v11-02c simulations use previously regridded $2^{\circ} \times 2.5^{\circ}$ and $4^{\circ} \times 5^{\circ}$ meteorological fields, while the GCHP v11-02c simulations regrid the $0.25^{\circ} \times$ $0.3125^{\circ}$ fields to the cubed sphere on the fly through ExtData. A native-resolution cubed-sphere GEOS data output stream is presently under development at GMAO and will benefit GCHP by reducing the need for regridding.

All simulations were conducted on the Harvard Odyssey computational cluster. Both GCHP and GCC were compiled using the Intel Fortran compiler (v15.0.0), and MPI capabilities for GCHP were provided by OpenMPI (v1.10.3). Each node of the cluster has 32 Intel Broadwell $2.1 \mathrm{GHz}$ cores sharing $128 \mathrm{~GB}$ of RAM, and all nodes are connected via Mellanox FDR Infiniband. Input and output data are stored using a Lustre parallel file system, accessible through the same Infiniband network fabric. All simulations were scheduled to enforce exclusive access to the nodes, preventing possible performance degradation due to sharing of node resources.

Figure 3 shows the total time taken to perform the simulation at each resolution, both in terms of wall time and in terms of the time per 1000 simulated atmospheric columns in the model grid. Results using the conventional GCC sharedmemory platform at $4^{\circ} \times 5^{\circ}$ and $2^{\circ} \times 2.5^{\circ}$ resolutions are also shown for comparison.

At the lowest simulated resolution (C24), GCHP's runtime exceeds that of GEOS-Chem Classic. This is predominantly due to overhead associated with file open operations, as the native-resolution meteorological data used to drive GCHP are opened and read independently for each field. This effect is clearly visible in the lower plot of Fig. 3, where performance penalties due to this overhead result in significantly longer runtimes per 1000 columns at C24 compared to C180. This can be addressed in the future through both structural changes and parallelization of the input.

After doubling the resolution to $\mathrm{C} 48\left(2^{\circ} \times 2.5^{\circ}\right)$, GCHP begins to outperform GCC, with a reduced overall simulation time even at core counts which are currently accessible to GCC, despite the larger input requirements of GCHP. A 1-month simulation at $C 48$ requires only $6 \mathrm{~h}$ using 96 cores. GCHP scalability also improves as the model resolution increases. At C180, the reduction in simulation time for each doubling in the number of cores is approximately a factor of 1.6.

Two factors affect GCHP scalability: fixed costs and overhead. Fixed costs are for operations which run on a fixed number of cores, regardless of the number of cores dedicated to the simulation. Overhead is the need for additional coordination and data communication between cores, which grows with the number of cores. Eventually, the overhead of the additional cores exceeds the computational benefit, resulting in a performance plateau. This overhead includes one-off costs, such as the MPI interface initialization, which can be significant when running with a large number of cores but which can be reduced in relative terms by running longer simulations.

The scalability of each model component is shown in Fig. 4. This shows the total time spent on each component at $\mathrm{C} 48$ and $\mathrm{C} 180$ resolution as a function of the total number of cores used, from 6 cores up to 540 . We see that the dominant fixed cost at both $\mathrm{C} 48$ and $\mathrm{C} 180$ is input, which also dominates the overall cost for $\mathrm{C} 48$ with more than 48 cores. This is due to the serial nature of the current input code, overhead associated with file open operations, and the aforementioned use of native-resolution meteorological data for even low-resolution GCHP simulations. Output operations are a second fixed cost, being handled by a single core at all times. For these simulations, 22 3-D fields were stored with hourly frequency, and output was a minor contributor to overall costs. Fixed costs can be converted into scalable costs by parallelizing the component in question, and this is a future work agenda. 

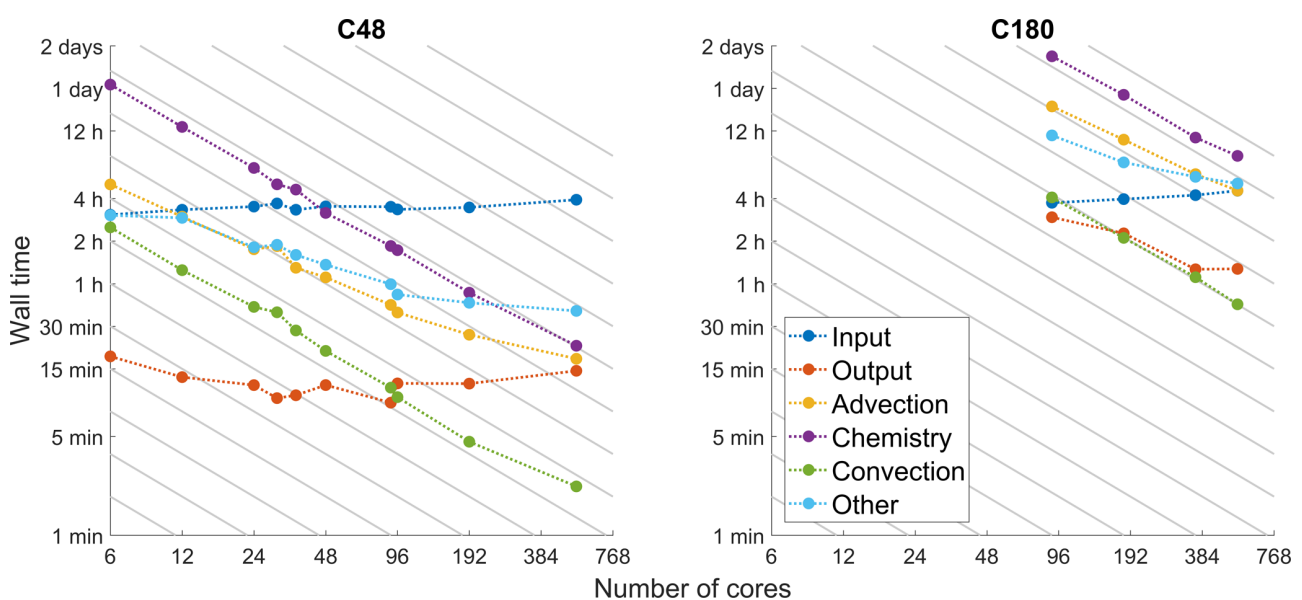

Figure 4. Total wall time per component for low (C48) and high (C180) resolution simulations. Simulations at C180 are limited to core counts of 90 or more across several nodes for the hardware used here, due to the high memory requirements of such high-resolution simulations.
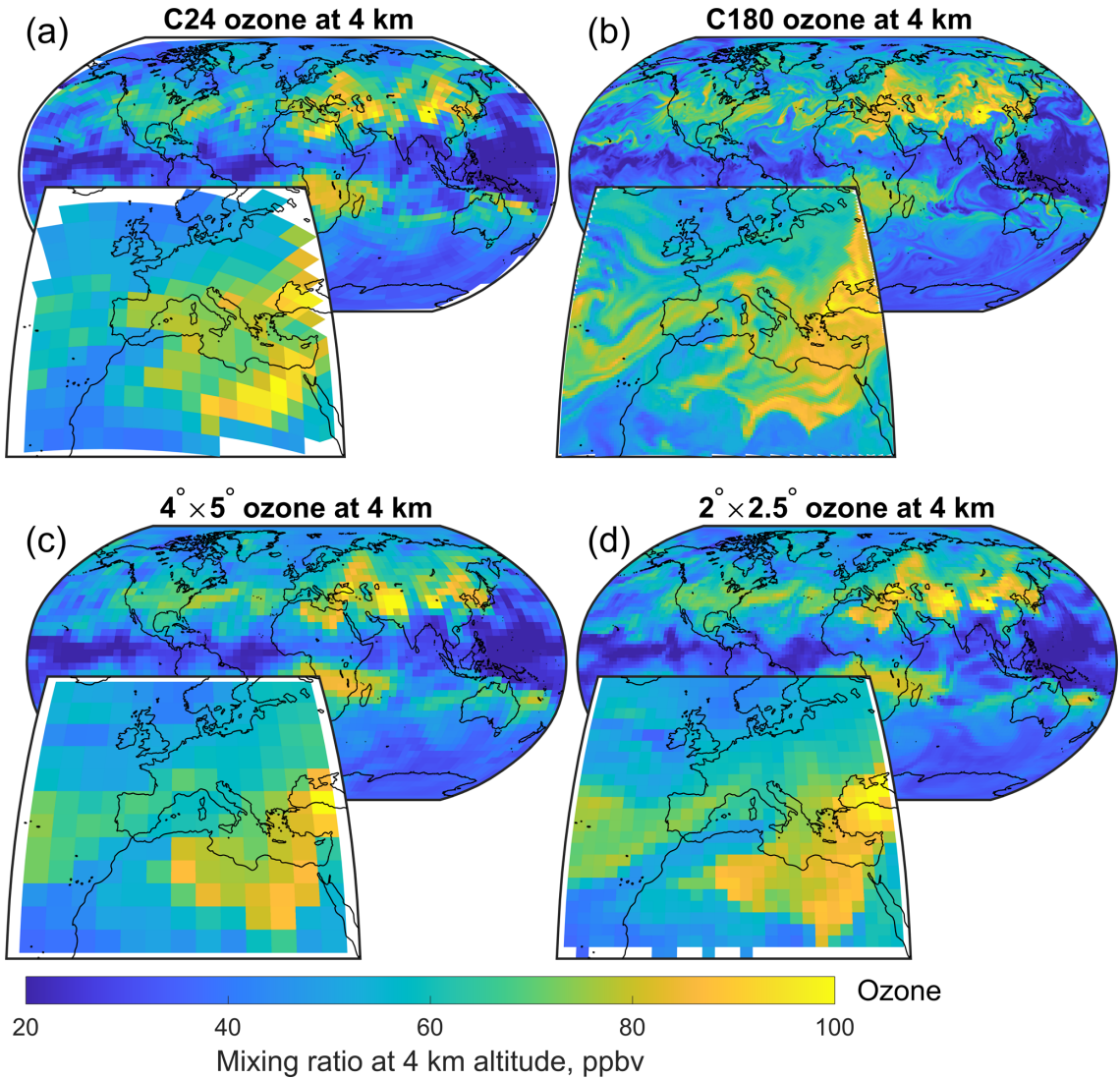

Figure 5. Simulated ozone concentrations at $4 \mathrm{~km}$ altitude for 23:00 UTC on 31 July 2016 after 1 month of initialization. The upper panels show model output from GCHP simulations at C24 (a) and C180 (b), while panels (c) and (d) show model output from GCC simulations at $4^{\circ} \times 5^{\circ}$ and $2^{\circ} \times 2.5^{\circ}$. Calculated values in some regions exceed the displayed limits. Zoom panels are also shown for Europe.

Chemistry, advection, and convection all scale well with increasing core count. Chemistry is the most expensive process at both coarse $(\mathrm{C} 48)$ and fine $(\mathrm{C} 180)$ resolution but has near-perfect scalability. Thus, at $\mathrm{C} 48$, we see that input becomes the limiting process when the number of cores ex- ceeds 48. Advection and other processes show more departure from perfect scalability, and may dominate the time requirement as the number of cores exceeds 600 . The scalability of advection suffers from the additional communication overhead associated with reducing the domain size, as each 

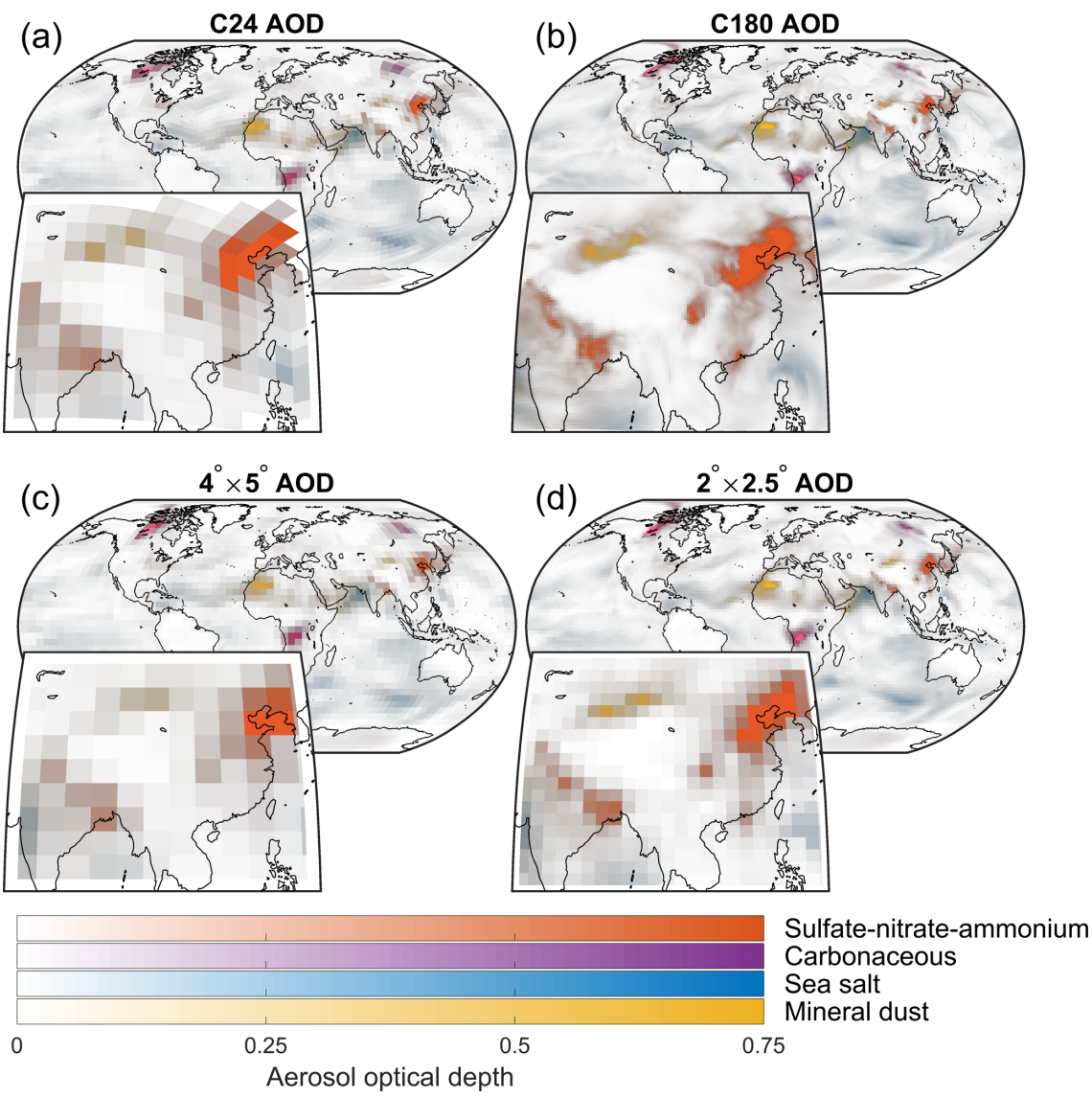

Figure 6. As for Fig. 5 but now showing daily average aerosol optical depth for 31 July 2016, after 1 month of initialization.

domain must communicate a larger proportion of its concentration data to its neighbors. More time is spent on communication relative to computation. However, wall time for advection does consistently fall with increasing core counts, an improvement compared to Long et al. (2015), where wall time increased as core counts exceeded 200 for a grid resolution equivalent to $\mathrm{C} 48$. We attribute this to the change from a latitude-longitude grid to the more scalable cubed-sphere grid.

The remaining wall time is taken up by the "other" component, a mix of scalable and non-scalable processes. This includes the one-off cost of initializing the MPI interface, which grows non-linearly with the number of cores. At C180, these costs are still exceeded by scalable costs when running with 540 cores, so no plateau in performance is observed.

\section{High-resolution simulations with GCHP}

The primary advantage of GCHP is the ability to perform simulations of atmospheric chemistry at resolutions previously not available to the community. Figure 5 shows illustrative distributions of simulated ozone concentration at $4 \mathrm{~km}$ altitude, simulated at $\mathrm{C} 24\left(\approx 4^{\circ} \times 5^{\circ}\right)$ and $\mathrm{C} 180(\approx$ $\left.0.5^{\circ} \times 0.625^{\circ}\right)$. Simulations were performed using 24 and 360 cores, respectively. Results are also shown from simulations using GCC, at $4^{\circ} \times 5^{\circ}$ and $2^{\circ} \times 2.5^{\circ}$. Emissions are identical for all simulations.

Global-scale patterns in ozone concentration are not substantially affected by the increase in resolution, as these are determined by large-scale processes. The agreement between the two simulations illustrates the consistency of GCHP across scales. However, increasing the horizontal resolution improves the ability of the model to capture the behavior of intercontinental plumes (Eastham and Jacob, 2017). The consequences of this are visible in the ozone distributions over the Pacific and Atlantic. We also observe maxima in the coarse-resolution simulation which are not visible in the finer-resolution simulation, such as the peak in ozone concentration over Egypt. This suggests possible simulation biases at coarse resolution.

Increasing the horizontal resolution also improves the ability of the model to resolve features at the scale of local air quality, as demonstrated by the plots of daily average aerosol optical depth (AOD) shown in Fig. 6. This is especially evident in the simulated column AOD over northern India and Beijing. For both ozone and AOD, comparison to the results from $\mathrm{GCC}$ at $4^{\circ} \times 5^{\circ}$ shows that simulations with 


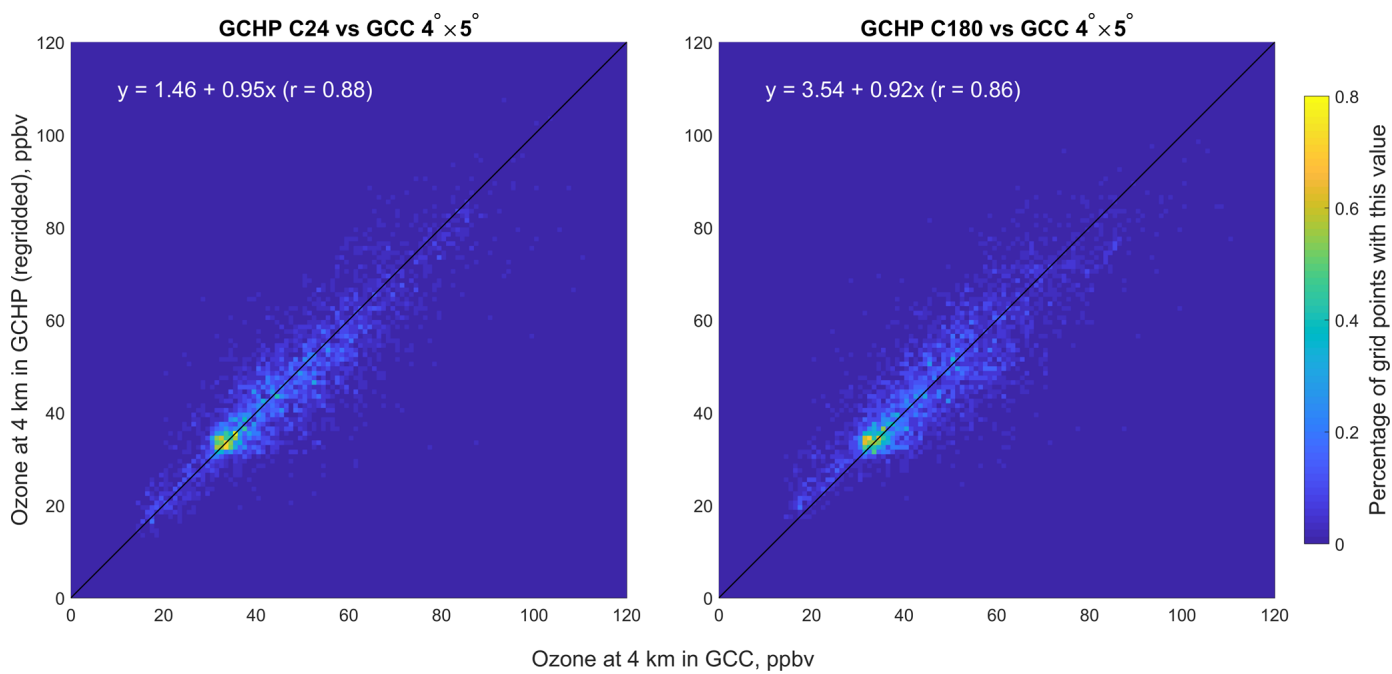

Figure 7. 2-D histograms of simulated ozone at $4 \mathrm{~km}$ altitude 23:00 UTC on 31 July 2016 after 1 month of initialization as calculated by GCC and GCHP. Each panel compares the simulated output from GCHP at a specific resolution with the simulated output from GCC at $4^{\circ} \times 5^{\circ}$. Data are binned at a resolution of 1 ppbv. GCHP data are conservatively regridded to $4^{\circ} \times 5^{\circ}$ prior to comparison. Data in the top and bottom two latitude bands are excluded, as GCC averages these points into two "polar caps".

GCHP at coarse (C24) resolution are able to reproduce the same patterns, magnitude, and variability as those observed in GCC. Some differences are observed, such as the region over Afghanistan which shows an elevated ozone mixing ratio in GCC compared to GCHP. However, the results from GCHP at $\mathrm{C} 24$ show the same patterns, magnitude, and variability as those at $4^{\circ} \times 5^{\circ}$.

Finally, we directly compare the simulated ozone data from GCHP and GCC using 2-D histograms. Figure 6 shows the ozone ratio in each $4^{\circ} \times 5^{\circ}$ grid cell, as simulated in GCC (horizontal axis) and as simulated in GCHP (vertical axis) at two different horizontal resolutions. All data are binned at a resolution of 1 ppbv ozone. In each case, GCHP results are conservatively gridded to $4^{\circ} \times 5^{\circ}$ to provide a direct comparison, and data in the "polar cap" regions (top and bottom two $4^{\circ} \times 5^{\circ}$ latitude bands) are excluded. Fitting parameters are shown in white based on a reduced-major-axis ("geometric") regression. Comparison of results at $\mathrm{C} 24$ to those at $4^{\circ} \times 5^{\circ}$ shows good agreement, with a correlation coefficient $r=0.88$ and a slope of 0.95 . At higher resolution, this agreement is slightly worsened $(r=0.86$ and slope of 0.92 ), as smaller-scale processes and chemical non-linearity are resolved which could not be represented at the coarser resolution.

\section{Summary}

Models of atmospheric chemistry have grown continuously in resolution and complexity over the past decades to take advantage of increasing computational resources. The GEOSChem High Performance (GCHP) model is a next step in this growth, enabling the widely used GEOS-Chem chem- ical transport model to exploit the computational speed and memory capacity of massively parallel architectures. In this manner, we can achieve routine simulation of global stratosphere-troposphere oxidant-aerosol chemistry at unprecedented resolution and detail.

Detailed documentation of GCHP including a user manual is available on the GCHP website (http://www.geos-chem. org, last access: 19 July 2018). GCHP incorporates the existing GEOS-Chem shared-memory code into an ESMF-based framework (MAPL), enabling GEOS-Chem to be run in a distributed memory framework across multiple nodes while retaining all the features of the high-fidelity global chemical simulation. In addition to a new model framework, GCHP replaces the conventional rectilinear latitude-longitude grid with the gnomonic cubed-sphere grid of the NASA GEOS meteorological data used as input to GEOS-Chem. This provides greater computational accuracy and efficiency for transport calculations while removing an additional restriction on scalability. GCHP performs with high computational scalability up to at least 540 cores, completing a 1-month simulation of oxidant-aerosol chemistry in the troposphere and stratosphere (206 active species, 135 tracers) at a global resolution of $\mathrm{C} 180\left(\sim 0.5^{\circ} \times 0.625^{\circ}\right)$ in $24 \mathrm{~h}$.

GCHP also provides a mechanism for ongoing improvement of modeling capability. With the base GEOS-Chem model, GCHP, and the GMAO GEOS atmospheric data assimilation system now all using an identical copy of the gridindependent GEOS-Chem code, GCHP closes the loop between online and offline modelers, allowing seamless propagation of model and framework improvements between all three. Future development opportunities range from improved parallelism in input operations to the direct ingestion 
of archived mass fluxes to further improve transport calculation accuracy.

Code availability. GCHP has been openly available as part of the GEOS-Chem code since beta version release v11-02b in June 2017, and was part of the v11-02 public release in March 2018. Complete documentation and access to the GCHP code can be found at http://www.geos-chem.org (last access: 19 July 2018). GCHP is an added functionality for GEOS-Chem users, who can choose to use either GCC or GCHP from the same code download. Both GCC and GCHP functionalities will be maintained in the standard GEOSChem model for the foreseeable future, recognizing that many users may not have access to the resources needed to use GCHP. For this work, GCHP v11-02c was used, a copy of which has been permanently archived (https://doi.org/10.5281/zenodo.1290835; Eastham et al., 2018). 
Appendix A: Acronyms used in this paper

$\begin{array}{ll}\text { Acronym } & \text { Description } \\ \text { AOD } & \text { Aerosol optical depth } \\ \text { CFL } & \text { Courant-Friedrichs-Lewy number } \\ \text { CTM } & \text { Chemical transport model } \\ \text { DAS } & \text { Data assimilation system } \\ \text { ESMF } & \text { Earth System Modeling Framework } \\ \text { FV3 } & \text { Finite-Volume Cubed-Sphere Dynamical Core } \\ \text { GCC } & \text { GEOS-Chem Classic } \\ \text { GCHP } & \text { GEOS-Chem High Performance } \\ \text { GCM } & \text { Global climate model } \\ \text { GEOS } & \text { Goddard Earth Observation System } \\ \text { GMAO } & \text { NASA Global Modeling and Assimilation Office } \\ \text { HEMCO } & \text { Harvard-NASA Emissions COmponent } \\ \text { MAPL } & \text { Modeling and Analysis Prediction Layer } \\ \text { MPI } & \text { Message Passing Interface } \\ \text { UCX } & \text { Unified Chemistry eXtension }\end{array}$


Author contributions. MSL and AM designed the initial code infrastructure. RVM and DJJ provided project oversight and top-level design. SDE, MSL, CAK, EWL, MY, RY, JZ, MT, AT, TC, JK, WP, and CJL performed code development.

SDE, EL, RVM, CL, and CJL adapted the meteorological archive. SDE, MSL, EWL, and JZ ran and debugged benchmark simulations. SDE, MSL, RY, and JZ performed scalability testing analysis. SDE, MSL, CAK, RY, EWL, JZ, RVM, and DJJ wrote the manuscript. All authors contributed to manuscript editing and revisions.

Competing interests. The authors declare that they have no conflict of interest.

Acknowledgements. This work was supported by the NASA Atmospheric Composition Modeling and Analysis Program (ACMAP) and the NASA Modeling, Analysis and Prediction (MAP) program. Sebastian D. Eastham was supported by the NOAA Climate and Global Change Postdoctoral Fellowship Program, administered by UCAR's Visiting Scientist Programs. Sebastian D. Eastham was also supported by a Harvard University Center for the Environment (HUCE) Postdoctoral Fellowship. The GEOS FP data used in this study/project were provided by the Global Modeling and Assimilation Office (GMAO) at NASA Goddard Space Flight Center. The computations were run on the Odyssey cluster supported by the FAS Division of Science, Research Computing Group at Harvard University. We are grateful to Compute Canada for hosting the data portal for GEOS-Chem to store and make available the GEOS meteorological fields used here. The authors would also like to thank Kevin Bowman for providing analytical insight and additional computational resources with which to perform early testing of the GCHP model. Finally, we would like to thank Junwei $\mathrm{Xu}$ for assistance in processing and archiving meteorological data for input to the model.

Edited by: Gerd A. Folberth

Reviewed by: two anonymous referees

\section{References}

Bey, I., Jacob, D. J., Yantosca, R. M., Logan, J. a., Field, B. D., Fiore, A. M., Li, Q., Liu, H. Y., Mickley, L. J., and Schultz, M. G.: Global modeling of tropospheric chemistry with assimilated meteorology: Model description and evaluation, J. Geophys. Res., 106, 23073, https://doi.org/10.1029/2001JD000807, 2001.

Brasseur, G. P. and Jacob, D. K.: Modeling of Atmospheric Chemistry, Cambridge University Press, https://doi.org/10.1017/9781316544754, 2017.

Eastham, S. D. and Jacob, D. J.: Limits on the ability of global Eulerian models to resolve intercontinental transport of chemical plumes, Atmos. Chem. Phys., 17, 2543-2553, https://doi.org/10.5194/acp-17-2543-2017, 2017.

Eastham, S. D., Weisenstein, D. K., and Barrett, S. R. H.: Development and evaluation of the unified tropospheric-stratospheric chemistry extension (UCX) for the global chemistry-transport model GEOS-Chem, Atmos. Environ., 89, 52-63, 2014.

Eastham, S. D., Long, M. S., Keller, C. A., Lundgren, E.h, Yantosca, R. M., Zhuang, J., and Jacob, D. J.: sdeastham/GCHP_v11-02c_Paper: GCHP v11-02c (Version v11-02c), Zenodo, https://doi.org/10.5281/zenodo.1290835, last access: 19 July 2018.

Fairlie, D. T., Jacob, D. J., and Park, R. J.: The impact of transpacific transport of mineral dust in the United States, Atmos. Environ., 41, 1251-1266, 2007.

Hill, C., DeLuca, C., Balaji, Suarez, M., and Silva, A. D.: The architecture of the Earth System Modeling Framework, Comput. Sci. Eng., 6, 18-28, 2004.

Horowitz, L. W., Walters, S., Mauzerall, D. L., Emmons, L. K., Rasch, P. J., Granier, C., Tie, X., Lamarque, J.-F., Schultz, M. G., Tyndall, G. S., Orlando, J. J., and Brasseru, G. P.: A global simulation of tropospheric ozone and related tracers: Description and evaluation of MOZART, version 2, J. Geophys. Res., 108, D24, https://doi.org/10.1029/2002JD002853, 2003.

Hu, L., Keller, C. A., Long, M. S., Sherwen, T., Auer, B., Da Silva, A., Nielsen, J. E., Pawson, S., Thompson, M. A., Trayanov, A. L., Travis, K. R., Grange, S. K., Evans, M. J., and Jacob, D. J.: Global simulation of tropospheric chemistry at $12.5 \mathrm{~km}$ resolution: performance and evaluation of the GEOSChem chemical module (v10-1) within the NASA GEOS Earth System Model (GEOS-5 ESM), Geosci. Model Dev. Discuss., https://doi.org/10.5194/gmd-2018-111, in review, 2018.

Jöckel, P., von Kuhlmann, R., Lawrence, M. G., Steil, B., Brenninkmeijer, C. A. M., Crutzen, P. J., Rasch, P. J., and Eaton, B.: On a fundamental problem in implementing flux-form advection schemes for tracer transport in 3-dimensional general circulation and chemistry transport models, Q. J. Roy. Meteor. Soc., 127, 1035-1052, 2001.

Keller, C. A., Long, M. S., Yantosca, R. M., Da Silva, A. M., Pawson, S., and Jacob, D. J.: HEMCO v1.0: a versatile, ESMF-compliant component for calculating emissions in atmospheric models, Geosci. Model Dev., 7, 1409-1417, https://doi.org/10.5194/gmd-7-1409-2014, 2014.

Lin, J.-T. and McElroy, M. B.: Impacts of boundary layer mixing on pollutant vertical profiles in the lower troposphere: Implications to satellite remote sensing, Atmos. Environ., 44, 17261739, 2010.

Lin, S.-J.: A "Vertically Lagrangian" Finite-Volume Dynamical Core for Global Models, Mon. Weather Rev., 132, 2293-2307, 2004.

Liu, H., Jacob, D. J., Bey, I., and Yantosca, R. M.: Constraints from $210 \mathrm{~Pb}$ and $7 \mathrm{Be}$ on wet deposition and transport in a global threedimensional chemical tracer model driven by assimilated meteorological fields, J. Geophys. Res., 106, 12109-12128, 2001.

Long, M. S., Yantosca, R., Nielsen, J. E., Keller, C. A., da Silva, A., Sulprizio, M. P., Pawson, S., and Jacob, D. J.: Development of a grid-independent GEOS-Chem chemical transport model (v9-02) as an atmospheric chemistry module for Earth system models, Geosci. Model Dev., 8, 595-602, https://doi.org/10.5194/gmd-8-595-2015, 2015.

National Research Council: A National Strategy for Advancing Climate Modeling, The National Academies Press, Washington, DC, 2012. 
Nielsen, J. E., Pawson, S., Molod, A., Auer, B., da Silva, A. M., Douglass, A. R., Duncan, B., Liang, Q., Manyin, M., Oman, L. D., Putman, W., Strahan, S. E., and Wargan, K.: Chemical Mechanisms and Their Applications in the Goddard Earth Observing System (GEOS) Earth System Model, J. Adv. Model. Earth Sy., 9, 3019-3044, 2017.

Prather, M. J.: Fast-JX v7.0a, available at: https://www.ess.uci.edu/ group/prather/scholar_software (last access: 19 July 2018), 2012.

Putman, W. M. and Lin, S. J.: Finite-volume transport on various cubed-sphere grids, J. Comput. Phys., 227, 55-78, 2007.

Sherwen, T., Evans, M. J., Carpenter, L. J., Andrews, S. J., Lidster, R. T., Dix, B., Koenig, T. K., Sinreich, R., Ortega, I., Volkamer, R., Saiz-Lopez, A., Prados-Roman, C., Mahajan, A. S., and Ordóñez, C.: Iodine's impact on tropospheric oxidants: a global model study in GEOS-Chem, Atmos. Chem. Phys., 16, 11611186, https://doi.org/10.5194/acp-16-1161-2016, 2016.
Suarez, M., Trayanov, A., Hill, C., Schopf, P., and Vikhliaev, Y.: MAPL: A High-level Programming Paradigm to Support More Rapid and Robust Encoding of Hierarchical Trees of Interacting High-performance Components, in: Proceedings of the 2007 Symposium on Component and Framework Technology in Highperformance and Scientific Computing, ACM, New York, NY, USA, 11-20, 2007.

Ullrich, P. A. and Taylor, M. A.: Arbitrary-Order Conservative and Consistent Remapping and a Theory of Linear Maps: Part I, Mon. Weather Rev., 143, 2419-2440, 2015.

Wang, Y., Jacob, D. J., and Logan, J. A.: Global simulation of tropospheric $\mathrm{O}_{3}$-NOx-hydrocarbon chemistry: 1. Model formulation, J. Geophys. Res., 103, 10713-10725, 1998.

Wu, S., Mickley, L. J., Jacob, D. J., Logan, J. A., Yantosca, R. M., and Rind, D.: Why are there large differences between models in global budgets of tropospheric ozone?, J. Geophys. Res., 112, D05302, https://doi.org/10.1029/2006JD007801, 2007. 